\title{
Liver Iron Concentration Assessed by SQUID Biosusceptometry Compared to Heat-dried Liver Biopsy: A Blinded Study.
}

Marcela Weyhmiller ( $\square$ marcela.gonzales@gmail.com )

UCSF Benioff Children's Hospital

\section{Zahra Pakbaz}

UCSF Benioff Children's Hospital

John Butz

Mayo Clinic

Ellen Fung

UCSF Benioff Children's Hospital

Douglas Paulson

Tristan Technologies (United States)

Kevin Pratt

Tristan Technologies (United States)

Jon Rowland

UCSF Benioff Children's Hospital

Elliott Vichinsky

UCSF Benioff Children's Hospital

Ashutosh Lal

UCSF Benioff Children's Hospital

Roland Fischer

University Medical Center Hamburg-Eppendorf

Paul Harmatz

UCSF Benioff Children's Hospital

\section{Research Article}

Keywords: Biomagnetic liver susceptometry (BLS), LIC, independent measures, SQUID, fresh tissue

Posted Date: December 6th, 2021

DOl: https://doi.org/10.21203/rs.3.rs-1135845/v1 
License: (c) (i) This work is licensed under a Creative Commons Attribution 4.0 International License. Read Full License 


\section{Abstract}

\section{Objective}

Biomagnetic liver susceptometry (BLS) is a noninvasive method to quantify liver iron concentration (LIC). Here we report our findings from a prospective study which validates in vivo LIC from a SQUID biosusceptometer by in vitro LIC in fresh tissue and paraffin-embedded biopsies from patients at risk for iron overload.

\section{Materials and Methods}

LIC was measured by BLS and biopsy. LIC by biopsy were measured in 40 dry weight fresh tissue and paraffin-embedded liver biopsy samples. LIC from biopsies and total iron scores from histology were compared to biosusceptometry. In addition, the wet-to-dry weight ratio was determined.

\section{Results}

Liver iron concentrations measured by BLS and in 40 fresh tissue biopsies were related by a factor of 6.0 $\pm 0.2\left(r^{2}=0.88\right)$. Similar results were obtained from comparisons with deparaffinized biopsies $(6.6 \pm 0.3$, $\left.r^{2}=0.87\right)$ and histology $\left(6.7 \pm 1.3, r^{2}=0.47\right)$. In contrast, a mean wet-to-dry weight ratio of $4.1 \pm 0.7$ was achieved from biopsies immediately weighed after the biopsy procedure.

\section{Conclusion}

LIC derived from two independent measures, the historical biopsy gold standard and biosusceptometry, were highly correlated. When comparing biosusceptometry with wet weight biopsies, the liver tissue sample size is critical.

\section{Introduction}

Patients with iron overload due to chronic blood transfusion (thalassemia, sickle cell disease) or increased iron absorption (hereditary hemochromatosis), are at risk for iron toxicity. If not adequately treated by iron depleting therapies (chelators, phlebotomy), patients can develop multiorgan failure: including liver cirrhosis, endocrinopathy, or cardiac failure ${ }^{1}$. Serum ferritin is widely used to monitor iron stores. Although it may correlate with liver iron concentration (LIC), when used alone, serum ferritin can be misleading ${ }^{2}$. Total body iron stores are reflected by liver iron concentration (LIC) $)^{3,4}$, therefore, LIC is routinely assessed to monitor iron overload and response to iron depletion therapies. Chemical quantification of iron in liver tissue samples collected by biopsy was originally used to monitor iron overload. Frequency of biopsy is limited by cost, risk and invasiveness, therefore noninvasive methods have been developed to allow for more frequent LIC assessment. In 1967, Bauman and Harris demonstrated that the difference between the magnetic susceptibilities of water in the body's tissues and the hemosiderin/ferritin iron complex in the liver could be used to quantify LIC ${ }^{5}$. Since then, several 
groups explored the application of biomagnetic liver susceptometry (BLS) technology using lowtemperature (liquid helium) SQUID (Superconducting QUantum Interference Device) biosusceptometers for the noninvasive quantification of LIC in humans ${ }^{6-10}$. Although dedicated to measuring LIC, the widespread adoption of SQUID-based technology was prevented by high operating costs, low environmental magnetic noise requirements, and a certain level of operation expertise. In an attempt to overcome the challenges of low-temperature biosusceptometers, room-temperature based instrumentation or superconducting high-temperature (liquid nitrogen) are in various phases of development ${ }^{11-14}$.

Validation against biopsy, the historic "gold standard" for LIC assessment, is a crucial step in the development of noninvasive iron quantification technology. In order to make comparisons between techniques, it is important to consider fundamental differences in the state of the sample at the time of testing. Noninvasive techniques are in vivo regional assessments of LIC (typically, a 3-dimensional nearsurface volume of about $300 \mathrm{~cm}^{3}$ by BLS or a 2-dimensional 5-10 mm slice with magnetic resonance imaging (MRI)) where the liver is "hydrated" or wet. In contrast, biopsy measures a small (about 10-15 $\mathrm{mm}^{3}$ ) in vitro sample of tissue processed and dried prior to iron quantification. Researchers developing MRI techniques overcame this comparison issue by calibrating liver relaxation rates (R2, R2*) against iron concentration measured by autopsy and biopsy dry weight data ${ }^{15,16}$. LIC from MRI is reported as milligrams iron per gram liver dry weight and therefore is comparable to LIC from liver biopsy.

Because the BLS technique depends on physical properties of iron, biosusceptometers are calibrated using the magnetic susceptibility, $\chi$, of an standard object (phantom) with well-known geometry ${ }^{10,17}$. The derived LIC relies only on the specific magnetic susceptibility of the hemosiderin/ferritin iron complex. For BLS to be applied to the near-surface organs (liver, spleen) of humans, the anatomical liver geometry must be estimated separately, e.g., by sonography. The MRI technique of quantitative susceptibility mapping (QSM), which is currently under development for liver iron measurements, relies on the same physical principles as $B L S^{18}$. LIC from BLS is reported as microgram iron per gram liver wet weight, and not comparable to dry weight liver biopsy without a conversion factor. Initial studies of BLS suggested that the conversion factor $\left(f_{\text {wdr }}\right.$ ) should be equal to the wet-to-dry weight ratio (WDR) defined as the ratio of the sample weight sample before (wet) and after drying. Early work utilized a WDR of 3.46, which assumed a water content of $71.1 \%$ for the liver ${ }^{6}$. However, studies in iron overloaded patients have shown variability in the WDR obtained from biopsies suggesting that WDR may depend on the method of biopsy and the size of the sample obtained ${ }^{19}$. A systematic investigation of the conversion of the in vivo (wet) volumetric measurement of iron by BLS into the in vitro (dry) chemical analysis of iron in small tissue samples by biopsy has not been previously reported.

This blinded, prospective study validates in vivo LIC from a SQUID biosusceptometer by in vitro LIC from fresh tissue (FT) biopsies and widely used paraffin-embedded biopsies (PB). From the wet and dry weight of biopsy samples, we calculated the conversion factor needed to compare LIC from BLS and LIC from liver biopsy. Preliminary data were reported in $2006^{20}$. 


\section{Materials And Methods \\ Patients and methods}

This study was approved by the institutional review board (IRB) at UCSF Benioff Children's Hospital Oakland. The procedures used in this study adhere to the tenets of the Declaration of Helsinki.

Written informed consent was obtained from all patients, and/or their guardian prior to participation in the study.

Eighteen patients with sickle cell disease (SCD) and 21 patients with transfusion dependent thalassemia (TDT) who were referred for clinically indicated percutaneous liver biopsy for LIC assessment, participated in the study. All participants were on chronic blood transfusion and chelation therapy. Mean age was 19.6 years (range, $5-40$ y). Each participant underwent liver iron assessment by biomagnetic liver susceptometry (BLS) and liver biopsy. A total of 45 BLS measurements could be compared with the results from 40 fresh tissue and 42 paraffin-embedded biopsy samples analyzed at Mayo Medical Laboratories, Rochester, MN (MAYO). Six patients had a second biopsy $27 \pm 6$ months after the first one.

Hepatitis (B or C) viral infection status, ferritin, liver transaminase (ALT), collected closest to the biopsy date, were extracted from patients' medical records.

\section{Liver biopsy}

Spring-loaded cutting needles (16g Bard $®$ Monopty $®$ Bard Endoscopic Technologies, Billerica, MA) with a $15 \mathrm{~mm}^{3}$ groove for tissue uptake were used for the biopsy procedure and 2 cores were obtained in 2 passes aiming at the same local area of the right liver lobe. Systematically, the liver tissue from $1 \mathrm{st}$ biopsy pass was used for histology purposes (paraffın embedding) and later deparaffinized for iron quantitation at MAYO, while the liver tissue from 2nd biopsy pass was used as the fresh tissue sample for iron quantitation.

Fresh tissue liver biopsy samples were weighed (mean wet weight $10.9 \pm 2.2 \mathrm{mg}$ ) in pre-weighed trace element free vials (cryovials were acid-washed at our institution) and shipped to MAYO. The resulting net weight was used as tissue sample wet weight. Since a humidity loss on the biopsy needle groove could not be excluded due to water resorption, we determined the residual water on the needle tip after the biopsy procedure and after drying the tip. We found approximately $0.9 \mathrm{mg}$ of residual water on needle tips for an average wet weight of $10.9 \mathrm{mg}$ of the tissue samples. At MAYO, the samples were divided into 2 parts and heat-dried $\left(95^{\circ} \mathrm{C}\right.$ for at least 17 hours) resulting in a total mean dry weight of $2.7 \pm 0.7 \mathrm{mg}$ (range, $1.0-4.0 \mathrm{mg}$ ). Dry samples were digested and aliquots $(20 \mu \mathrm{l})$ were injected into an Inductively Coupled Plasma quadrupole Mass Spectrometer (ICP-MS: Elan DRC II®, Perkin-Elmer, Boston, USA) for iron measurement.

As described in detail by Butensky et al (2005) ${ }^{21}$, Scheuer and Deugnier scoring system was applied to histologic sections to assess inflammation, fibrosis and total iron scores (TIS). After obtaining sections 
for histology, paraffin-embedded liver samples were deparaffinized (mean dry weight $1.1 \pm 0.4 \mathrm{mg}$ ) according to the method described by Bush et $\mathrm{al}^{22}$. The imprecision of deparaffinized liver biopsy LIC values was estimated from the day-to-day variation of $7 \%$ as determined by analyzing NBS reference standards.

\section{Biosusceptometry}

Most patients (88\%) underwent SQUID biosusceptometry within a month post liver biopsy. The technical details and the measurement procedure of the biosusceptometer (Ferritometer ${ }^{\circledR}$, Model 5700, Tristan Technologies, San Diego, USA) were described elsewhere ${ }^{23,24}$. More recently, liver susceptometry was described in detail in relation to $\mathrm{MRI}^{25,26}$. In short, subjects were placed in a supine position on a motor driven bed with low magnetic signature. Bed-side sonography was used for assessment of the thorax, anterior liver geometry and measurement of the skin-liver distance. Subjects were centered at closest distance below the superconducting magnetic field (max. $35 \mathrm{mT})$ and the gradiometer detection coils. SQUID voltages, distance, position, and pressure signals were acquired during a 10 sec breath-hold vertical scan. The data were analyzed within an analytical model ${ }^{10}$ of magnetic flux integrals $\left(\Phi(z)=\int B_{f}\right.$ - $B_{d} d^{3} r$ ) calculated for ellipsoidal liver and cylindrical thorax geometries at distance $z$ resulting in equation (1).

(1) $\Delta \mathrm{V}=\mathrm{C}\left(\Delta \mathrm{\chi}_{\text {liver }} \cdot \boldsymbol{\Phi}_{\text {liver }}+\Delta \mathrm{\chi}_{\text {thorax }} \cdot \boldsymbol{\Phi}_{\text {thorax }}\right)+\Delta \mathrm{V}_{\text {system }}$

The system was calibrated (C) by an infinite water sphere versus air with the well-known magnetic susceptibility difference of $9.396 \cdot 10^{-6}$ [SI-units]. After subtraction of the magnetic contribution of the anterior thorax tissue ( $\left.\Delta \chi_{\text {thorax }}\right)$ calculated from the patients' body mass index (BMI), the in vivo liver iron concentration LIC is calculated from its magnetic volume susceptibility relative to water, $\Delta \mathrm{\chi}_{\text {liver, }}$, and the specific magnetic susceptibility of the hemosiderin-ferritin iron complex $\chi_{F e}\left(=1,524 \cdot 10^{-6}\right.$ [SI-units] including the liver tissue density $\rho\left(=1.05 \mathrm{~g} / \mathrm{cm}^{3}\right)^{27}$, equation (2).

(2) $\mathrm{LIC}=\Delta \mathrm{X}_{\text {liver }} /\left(\mathrm{X}_{\mathrm{Fe}} \cdot \rho\right) \cdot \mathrm{f}_{\mathrm{wdr}}$

To convert SQUID-BLS LIC from units of wet weight liver to dry weight the wet-to-dry weight conversion factor, $f_{w d r}$, has to be taken into account ${ }^{26}$.

Assessment of liver volume was performed according to the method of Leung et al ${ }^{28}$. In short, a $3.5 \mathrm{MHz}$ linear array ultrasound probe (length $10 \mathrm{~cm}$ ) was vertically aligned with a cross-plane laser over the liver of a patient in the supine position on the biosusceptometer bed. The bed allows transverse and longitudinal movements in scaled steps. The area of sagittal slices at incremental steps of $2 \mathrm{~cm}$ were determined in the trace mode by the imager software (EUB-500, Hitachi Med. Systems Inc., Tokyo, Japan). The total volume was calculated from the slice areas and thickness $(2 \mathrm{~cm})$.

\section{Data analysis and statistics}


In order to establish blindness during analysis of the biosusceptometry data, 31/40 biosusceptometry results were analyzed and sent to a data depository (Drug and Device Development Co., Inc., Redmond, WA, USA) prior to receiving the fresh tissue biopsy results from MAYO. Nine biopsy results were received before the results from BLS could be sent to the data depository. All biopsy procedures were performed between 0 and 58 days before biosusceptometry. In six patients, biopsies were repeated after 17 to 36 months. All results from paraffin-embedded biopsies were received after the BLS procedure.

To calculate the wet-to-dry weight conversion factor, $f_{w d r}$, a weighted linear fit (Marquardt algorithm) with no intercept was used as the most robust method. As both iron measurement methods suffer from their significant imprecisions, a statistical weight was calculated as sum of total uncertainties of BLS (1/

$\left.\sigma_{\mathrm{BLS}}{ }^{2}\right)$ and biopsy $\left(1 / \sigma_{\mathrm{Biopsy}}{ }^{2}\right)$. Total uncertainties for BLS were calculated from standard deviations (SD) of skin-liver distance measured by sonography, from the uncertainty of BMI derived thorax susceptibility, and from the SD of 5 scans (Fig. 1). A mean total uncertainty for BLS of $\sigma_{\mathrm{BLS}}=168 \mu \mathrm{g} / \mathrm{g}_{\text {liver }}$ (range, $49-$ $344 \mu \mathrm{g} / \mathrm{g}_{\text {liver }}$ ) was found. The variability of LIC from dry weight liver biopsies $\left(\sigma_{\text {Biopsy }}\right)$ ranged from 29 to $2,300 \mu \mathrm{g} / \mathrm{g}_{\mathrm{dry}} \mathrm{w}_{\mathrm{t}}$ which was obtained from the two liver tissue parts (> $1 \mathrm{mg}$ dry weight each).

The following software packages were used for analysis: regression, weighted linear, and nonlinear fits were performed by Slide Write Plus for Windows (Version 7, Advanced Graphics Software Inc., Encinitas, CA, USA); Spearman rank correlation $\left(r_{S}\right)$ tests and Bland-Altman plots used the EXCEL add-on Analyse-it (Analyse-it Software Ltd, Leeds, UK); all other calculations were performed by Microsoft EXCEL (Microsoft Corp., Seattle, USA).

\section{Results}

\section{Correlation between $\mathrm{LIC}_{\mathrm{BLS}} \mathrm{LIC}_{\mathrm{FT}}$ and ferritin}

The mean LIC from fresh tissue biopsies ( $\mathrm{LIC}_{\mathrm{FT}}$ ) was 12,981 $\pm 8,237 \mu \mathrm{g} / \mathrm{g}_{\text {dry wt }}$ (range, 1,854 - 32,864 $\mu \mathrm{g} / \mathrm{g}$ ). The mean LIC from BLS (LIC BLS) was $2,071 \pm 1,275 \mu \mathrm{g} / \mathrm{g}_{\text {liver }}$ (range, $403-4,858 \mu \mathrm{g} / \mathrm{g}$ ). The association between liver iron concentrations measured by SQUID biosusceptometry and fresh tissue biopsy was non-parametrically tested in 40 measurement procedures with 6 patients measured twice, resulting in a Spearman rank correlation coefficient of $r_{S}=0.90(p<0.0001)$.

Correlation of ferritin with $\mathrm{LIC}_{\mathrm{BLS}}$ and $\mathrm{LIC}_{\mathrm{FT}}$ yielded $\mathrm{r}_{\mathrm{S}}=0.60(\mathrm{p}<0.0001)$ and $\mathrm{r}_{\mathrm{S}}=0.51(\mathrm{p}=0.0009)$, respectively (data not shown). Median ferritin values were lower for patients with TDT $(2,377 \mu \mathrm{g} / \mathrm{L})$ than $\operatorname{SCD}(3,071 \mu \mathrm{g} / \mathrm{L})$, however no significant difference was observed for the correlation $\left(r_{S}=0.58\right.$ and 0.65$)$.

\section{LIC conversion factor for SQUID-BLS}

In Fig. 1, an unbiased (including patients with $\mathrm{BMI}>30 \mathrm{~kg} / \mathrm{m}^{2}$ ) regression analysis, with $\mathrm{LIC}_{\mathrm{FT}}$ as reference method, yielded a linear relationship with LIC from BLS: $\mathrm{LIC}_{\mathrm{BLS}}=(0.14 \pm 0.01) \cdot \mathrm{LIC}_{\mathrm{FT}}+(244 \pm$ $154)$ with $r^{2}=0.83$. Since no liver biopsies were performed on normal controls in this study, LIC results 
from biopsies analyzed at MAYO on samples collected from 30 non-transfused human subjects at autopsy $\left(822 \pm 491 \mu \mathrm{g} / \mathrm{g}_{\text {dry wt }}\right)^{22}$ and BLS measurements in 10 controls at our institution (300 \pm 241 $\left.\mu \mathrm{g} / \mathrm{g}_{\text {liver }}\right)^{29}$ were plotted in Fig. 1 to demonstrate the normal range.

Based on the uncertainties of BLS and biopsy, a weighted linear fit $\left(r^{2}=0.88\right)$ with an a priori zero intercept resulted in a wet-to-dry weight conversion factor, $f_{\text {wdr }}$, of $6.04 \pm 0.22\left(\mu \mathrm{g} / \mathrm{g}_{\text {dry wt }}\right) /\left(\mu \mathrm{g} / \mathrm{g}_{\text {liver }}\right)$ for heat-dried LIC from fresh tissue samples (Table 1). With this conversion factor, the agreement between $\mathrm{LIC}_{\mathrm{BLS}}$ and $\mathrm{LIC}_{\mathrm{FT}}$ was tested by Bland-Altman plots. The $95 \%$ limits of agreement between $\mathrm{LIC}_{\mathrm{BLS}}$ and $\mathrm{LIC}_{\mathrm{FT}}$ were found between $-61.8 \%(-7,368 \mu \mathrm{g} / \mathrm{g})$ and $57.7 \%(6,200 \mu \mathrm{g} / \mathrm{g})$ with a negligible zero bias of $-2 \%$ $(-584 \mu \mathrm{g} / \mathrm{g})$.

\section{Correlation between $\mathrm{LIC}_{\mathrm{BLS}}$ and $\mathrm{LIC}_{\mathrm{DP}}$}

Paraffin-embedded biopsies are typically available allowing both histology and quantification of iron. A direct comparison by Altman-Bland plots between $\mathrm{LIC}_{\mathrm{BLS}}$ and $\mathrm{LIC}$ from deparaffinized biopsies (LIC $\left.\mathrm{DP}_{\mathrm{P}}\right)$ revealed $95 \%$ limits of agreement of $-24 \%(4,118 \mu \mathrm{g} / \mathrm{g})$ to $28 \%(5,733 \mu \mathrm{g} / \mathrm{g})$ with a significant proportional bias of about $2 \%$ (slope $0.15 \pm 0.04, p=0.001$ ). The mean weight of 37 deparaffinized biopsies was $1.1 \pm$ $0.4 \mathrm{mg}$ (range, $0.4-2.2 \mathrm{mg}$ ) and mean $\mathrm{LIC}_{\mathrm{DP}}$ was $13,633 \mu \mathrm{g} / \mathrm{g}_{\mathrm{dry} \mathrm{w}_{\mathrm{t}}}$, ranging from 1,717 to $35,219 \mu \mathrm{g} / \mathrm{g}_{\mathrm{dry}}$ wt. For conversion of $\mathrm{LIC}_{\mathrm{BLS}}$ into $\mathrm{LIC}_{\mathrm{DP}}$, a weighted linear fit $\left(\mathrm{r}^{2}=0.87\right)$ resulted in a conversion factor of $6.60 \pm 0.25\left(\mu \mathrm{g} / \mathrm{g}_{\text {dry wt }}\right) /\left(\mu \mathrm{g} / \mathrm{g}_{\text {liver }}\right)($ Fig. 2$)$.

Beyond LIC from fresh tissue biopsy, the additional impact from BMI, ALT, fibrosis and inflammation scores, and hepatitis (B or $\mathrm{C}$ ) viral infection on the relationship with SQUID-BLS was considered by multiple linear regression. Only the fibrosis score had an additionally small impact on the bi-variate correlation of SQUID-BLS with LIC by biopsy only $\left(r^{2}=0.817\right)$ and with adding fibrosis score $\left(r^{2}=0.837\right.$, $\mathrm{p}$ (fibrosis) $=0.02$ ).

\section{Histology results}

As an independent comparison, total iron scores (TIS) from histologic slides were compared with LIC $\mathrm{BLS}_{\text {S }}$ (Fig. 3). An exponential saturation function of type TIS $=a_{0} \cdot\left[1-\exp \left(-\beta \cdot a_{1} \cdot{ }^{2} C_{B L S}\right)\right]$ was used to fit the data, where $B=1.2 \cdot 10^{-4}(\mu \mathrm{g} / \mathrm{g})^{-1}$ was adopted from Butensky et $\mathrm{al}^{21}$, resulting in the maximum TIS score $a_{0}=$ $35.1 \pm 2.8$ and the conversion factor $\mathrm{a}_{1}=6.7 \pm 1.3$.

In Table 1, the wet-to-dry weight conversion factors are summarized together with coefficients of determination from linear regression of LIC or TIS by biopsy with LIC by SQUID-BLS. No significant difference between LIC from fresh and deparaffinized tissue samples was observed (paired Wilcoxon test: $\mathrm{p}=0.5)$. 
Table 1

Summary of Conversion Factors. Conversion factors \pm uncertainty for dry weight

LIC from fresh tissue, paraffin embedded biopsies, and total iron scores (TIS) derived from weighted linear regression with LIC by LIC-BLS.

\begin{tabular}{|c|c|c|c|}
\hline Sample & $\mathbf{n}$ & $\begin{array}{l}\text { Conversion } \\
\text { Factor } \\
\left(\mu \mathrm{g} / \mathrm{g}_{\text {dry wt }}\right) /\left(\mu \mathrm{g} / \mathrm{g}_{\text {liver }}\right)\end{array}$ & $\begin{array}{l}\text { Coefficient of } \\
\text { determination } r^{2}\end{array}$ \\
\hline fresh tissue biopsy $\left(\mathrm{LIC}_{\mathrm{FT}}\right)$ & 40 & $6.04 \pm 0.22$ & 0.88 \\
\hline deparaffınized $\left(\mathrm{LIC}_{\mathrm{DP}}\right)$ & 42 & $6.60 \pm 0.25$ & 0.87 \\
\hline TIS (histologic sections) & 44 & $6.7 \pm 1.3$ & 0.47 \\
\hline
\end{tabular}

A mean wet-to-dry weight ratio (WDR) of $4.1 \pm 0.7$ was obtained from the ratio of net weight of the freshtissue samples immediately after the biopsy procedure (wet weight) and the dry weight after the heating procedure at MAYO. However, in an intra-individual comparison (repeated biopsies) the WDR was not constant. In 6 patients, biopsies were repeated after 17 to 36 months. In contrast to an expected constant ratio of 1.0 between the WDR of the 1st and 2 nd biopsy, it varied between 0.63 and 1.28 in the respective biopsy pairs (Fig. 4, insert). The highest discrepancy (ratio 0.63 ) was related to hepatitis reactivity with inflammation score of 1 and 2.

Liver volumes (range, $666-3,018 \mathrm{~mL}$ ) were assessed by sonographic scanning. Comparing measured ultrasound volumes $\left(V_{u s}\right)$ to normal volumes $\left(V_{n o r}\right)$, calculated from Rasmussen ${ }^{30}$ by the respective body surface areas, the experimental WDR could be transformed into liver volume corrected ratios by: WDR* $=$ $1.1 \bullet \mathrm{V}_{\mathrm{us}} / \mathrm{V}_{\text {nor }} \bullet \mathrm{WDR}$. This also takes a humidity loss of $10 \%$ on the biopsy needle into account. The mean value for WDR of $4.1 \pm 0.7$ increased to a mean for WDR* of $5.9 \pm 1.9$, typically, with the largest value (13.1) for the maximum liver volume of $3,018 \mathrm{ml}$. Additionally, the significant correlation of WDR with the biopsy dry weight $\left(r_{S}=-0.52, p<.0001\right)$ became insignificant for WDR* $\left(r_{S}=-0.11\right)$, (Fig. 4).

\section{Discussion}

\section{BLS and biopsy}

In comparing the results from biosusceptometry and biopsy, we took into account the potential error of biosusceptometry, as well as that of biopsy. The correlation between LIC by BLS and fresh tissue biopsy, including 5 patients with $\mathrm{BMI}>30 \mathrm{~kg} / \mathrm{m}^{2}$, was good $\left(r_{S}=0.903\right)$ and did not significantly change upon exclusion of these patients $\left(r_{S}=0.906\right)$. Our results have a lower coefficient of determination $\left(r^{2}=0.88\right)$ than that found in a former study of SQUID-BLS versus LIC by wet weight biopsies from laparoscopy $\left(r^{2}=\right.$ 0.98 , range, $\left.30-4,840 \mu \mathrm{g} / \mathrm{g}_{\text {wet wt }}\right)^{9}$. However, in the previous study most patients were diagnosed with 
hereditary hemochromatosis and the mean biopsy weight was 50\% larger. A study, calibrating MRI-R2* with 23 biopsies in the same LIC range and using the same biopsy technique (fresh tissue, MAYO) gave better results $\left(r^{2}=0.94 \text {, Bland-Altman } 95 \% \text { agreement: }-46 \% \text { and } 44 \%\right)^{16}$. In another study, comparing MRI-R2 with LIC from freeze-dried biopsies a better correlation was achieved $\left(r_{S}=0.98\right)^{15}$. Prediction as characterized by coefficients of determination were also lower for BLS $\left(r^{2}=0.88\right)$ than for MRI-R2 $\left(r^{2}=\right.$ 0.96). However, we measured only 39 humans (6 were measured twice) and our LIC range was more limited ( $\leq 33 \mathrm{mg} / \mathrm{g}_{\text {dry wt }}$ ). Bland-Altman statistics yielded similar results for the $95 \%$ limits of agreement by MRI-R2 (-56\% and 50\%) and biosusceptometry (-62\% and 58\%) versus LIC by biopsy. The SQUID-BLS method was independent from calibration by biopsies, unlike MRI techniques, and could be the reason why comparisons between biopsy and MRI tend to have stronger correlations.

\section{Conversion of wet weight LIC to dry weight LIC}

Previously accepted work validating biosusceptometry by biopsy utilized a $f_{w d r}$ of 3.46 based on the water content of $71.1 \%$ of normal autopsy liver tissue $e^{6,31}$. Here, a conversion factor, $f_{w d r}$, of about 6 $\left(\mu \mathrm{g} / \mathrm{g}_{\text {dry wt }}\right) /\left(\mu \mathrm{g} / \mathrm{g}_{\text {liver }}\right)$ was calculated. This is consistent with other results from comparing LIC by noninvasive measurements to LIC from dry weight biopsies. In a study correlating LIC from dry weight biopsies with in vivo whole liver LIC assessed by a room-temperature based magnetic iron detector (MID) a wet-to-dry weight conversion factor $\left(f_{w d r}\right)$ of 5.8 was used to minimize the bias in an Altman-Bland plot $^{32}$. In a study comparing different MRI techniques, the magnetic hepatic susceptibility derived from the phase (field) difference between two adjacent small lateral ROls was compared with dry weight LIC from MRI-R2* measurements $\left(r^{2}=0.94\right)$ in the same vicinity of the liver ${ }^{33}$. Using a specific susceptibility

of $\chi_{\mathrm{Fe}}=1,450 \cdot 10^{-6}$ [SI-units] for liver iron (equation (2)) ${ }^{26}$, a conversion factor of $8.99 \pm 0.15 \mathrm{mg} \mathrm{Fe} / \mathrm{g}$ dry liver per $\mathrm{mg} \mathrm{Fe} / \mathrm{mL}$ wet liver was calculated, which is higher than our factor of $6\left(\mu \mathrm{g} / \mathrm{g}_{\mathrm{dry} \mathrm{wt}}\right) /\left(\mu \mathrm{g} / \mathrm{g}_{\text {liver }}\right)$. Understanding the basis of the difference between WDR and $\mathrm{f}_{\mathrm{wdr}}$ and their role in validation of the SQUIDBLS is the major significance of this work.

\section{Wet-to-dry weight ratio}

Current noninvasive iron measurement techniques are volumetric methods. The difference between WDR and $f_{w d r}$ calculated from the LIC results could be attributed to factors from the biopsy procedure. For biopsy, it is assumed that a representative volume is punched out of the liver. In this study, instead of the expected groove volume of $15 \mathrm{~mm}^{3}(15.7 \mathrm{mg}$ ) we obtained only a mean biopsy weight of $10.8 \mathrm{mg}$ using a cutting needle. In this aspect, the discrepancy between the expected sample weight (based on the groove volume of the biopsy needle) and the average weight of the tissue samples might be of interest since only $3 / 38$ biopsies had wet weights $>14 \mathrm{mg}$. In addition, the WDR of repeated biopsies varied between 1 and $40 \%$ for biopsies collected from the same patient at different times. Size of the biopsy could play a role in the WDR. Our mean WDR (4.1) is similar to a previous study of reference.

Zuyderhoudt et $\mathrm{al}^{34}$ found a mean percentage of $24.6 \%$ dry weight in wet weight biopsies (approximately $15 \mathrm{mg}$ ), i. e. a WDR of 4.1. However in the earlier study of Barry, the dry weight made up only $17 \%$ of large 
wet weight biopsies (> $5 \mathrm{mg}$ dry weight), which leads to a WDR of $5.9^{19}$. Later, a WDR of $5.5 \pm 0.9$ was found in large autopsy liver tissue samples (mean weight $68 \pm 50 \mathrm{~g}$ ) ${ }^{35}$. In a study comparing MRI-R2 with hepatic iron from 22 autopsy tissue samples $(100-500 \mathrm{mg})$ of non-human primates (marmosets), a WDR of $5.13 \pm 0.6$ was found ${ }^{36}$. One should expect a slightly smaller value of WDR than for true in vivo samples due to a potential blood loss during autopsy.

Liver volume may be important to consider when comparing biopsy with noninvasive measurements because liver volume can have an impact on WDR. Correcting the WDR for liver volume enlargement (hepatomegaly) resulted in a WDR* of $5.9 \pm 1.9$. However, there was no systematic trend in individual biopsies, i.e., enlarged liver volumes cause lower WDR in the same patient and vice versa. In addition, liver volume assessment by ultrasound may be less precise than assessment by MRI.

\section{Specific magnetic susceptibility}

All noninvasive methods rely on the magnetic susceptibility of the hemosiderin/ferritin iron complex. In a recent overview, specific susceptibilities for ferritin and/or hemosiderin were given in a range of (1,030$1,570) \cdot 10^{-6}$ [SI-units $]^{26}$. Total specific susceptibilities were obtained in the same range in spleen tissue from transfusion dependent and non-transfusion dependent thalassemia patients ${ }^{37}$. The current situation is characterized by LIC $\sim \mathrm{f}_{\mathrm{wdr}} / \chi_{\mathrm{Fe}}$ (see equation (2)), where $\mathrm{f}_{\mathrm{wdr}}$ may take values of 4 to 6 while $\chi_{\mathrm{Fe}}$ may take values between $1,050 \cdot 10^{-6}$ [SI-units] and 1,570 $10^{-6}$ [SI-units]. Variations in magnetic susceptibility will be reflected in the data from any magnetic method (also MRI) while variations in the biopsy desiccation procedure will counteract the final LIC relationship ${ }^{38}$.

\section{Conclusions}

In this paper, LIC assessed with the Model 5700 Ferritometer® was compared with biopsy in three ways, against wet weight, dry weight, and histologically. In order to make comparisons with MRI techniques, a conversion factor $\left(f_{w d r}\right)$ accounting for the difference between the wet and dry states of the liver at the time of assessment was taken into account. In this work, the wet-to-dry weight ratio (WDR) of 4.1 was not found to equal $f_{w d r}$, instead a factor of 6 was determined from fresh tissue and paraffin-embedded biopsies. This discrepancy may also be attributed to the small size of current liver biopsies. A very strong correlation was observed, thereby validating the Ferritometer ${ }^{\circledR}$ with the historical gold standard biopsy.

The ability to validate noninvasive techniques is critical for the development of novel technology, access to information for clinicians, and quality of life for patients. Patients at risk for iron overload require LIC assessment every 6 - 12 months, making biopsy impractical for clinical management. Many centers have switched to noninvasive magnetic techniques, either quantitative imaging by MRI or in a few centers biosusceptometry. MRI allows for the noninvasive assessment of iron for many tissues of interest. However, common MRI techniques still have some limitations. Relaxometry is limited to a maximum measurable LIC inversely proportional to magnetic field strength and many institutions are replacing 1.5 Tesla imagers with 3.0 Tesla technology roughly reducing maximum measurable LIC by half. In addition, 
small children may require sedation and intubation which increases cost and invasiveness. There is a continued need for the development and validation of novel noninvasive techniques for body iron assessment.

BLS is a promising supplement to MRI techniques. BLS provides an independent method for calibrating new quantitative magnetic resonance imaging techniques now that biopsy is rarely used exclusively for iron quantification. High-temperature and room-temperature biosusceptometers are being developed as noninvasive, cost effective, and potentially portable devices with potential for rapid analysis and high patient throughput ${ }^{11-14,39}$. These new BLS-based devices may meet the needs for noninvasive LIC measurements within communities with minimal resources across the globe. In addition, the ability to quantify LIC in small children without the need for sedation make these devices extremely valuable in a pediatric setting.

\section{Declarations}

\section{Data availability}

The datasets generated and analyzed during the current study are available from the corresponding author on reasonable request.

\section{Author information}

\section{Contributions}

MGW: study conception and design, analysis and interpretation of data, drafting of manuscript, critical revision. ZP: study conception and design, acquisition of data, drafting of manuscript, critical revision. JB: acquisition of data, analysis and interpretation of data. EF: study conception and design, drafting of manuscript, critical revision. DP: acquisition of data, drafting of manuscript. KP: acquisition of data, drafting of manuscript. JR: study conception and design, acquisition of data. EV: study conception and design, drafting of manuscript, critical revision. AL: critical revision. RF: study conception and design, acquisition of data, analysis and interpretation of data, drafting of manuscript, critical revision. $\mathrm{PH}$ : study conception and design, acquisition of data, analysis and interpretation of data, drafting of manuscript, critical revision. All authors have approved the submitted version and have agreed both to be personally accountable for the author's own contributions and to ensure that questions related to the accuracy or integrity of any part of the work, even ones in which the author was not personally involved, are appropriately investigated, resolved, and the resolution documented in the literature.

\section{Affiliations}

\section{UCSF Benioff Children's Hospital Oakland, Oakland, CA, USA}

M. G. Weyhmiller, Z. Pakbaz, E. Fung, J. Rowland, E. Vichinsky, A. Lal, R. Fischer \& P. Harmatz 
Mayo Medical Laboratories, Rochester, MN, USA

J. Butz

Tristan Technologies, San Diego, CA, USA

D. Paulson \& K. Pratt

Department of Diagnostic and Interventional Radiology, University Medical Center Hamburg-Eppendorf, Germany

R. Fischer

Corresponding authors

Correspondence to Marcela Weyhmiller and Paul Harmatz.

\section{Additional Information}

\section{Funding}

None.

\section{Ethics declarations}

\section{Competing Interests Statement}

All authors certify that they have no affiliations with or involvement in any organization or entity with any financial interest or non-financial interest in the subject matter or materials discussed in this manuscript.

\section{References}

1. Borgna-Pignatti, C. et al. Survival and complications in patients with thalassemia major treated with transfusion and deferoxamine., 89, 1187-1193 (2004).

2. Pakbaz, Z. et al. Serum ferritin underestimates liver iron concentration in transfusion independent thalassemia patients as compared to regularly transfused thalassemia and sickle cell patients. Pediatr. Blood Cancer, 49, 329-332 (2007).

3. Angelucci, E. et al. Hepatic iron concentration and total body iron stores in thalassemia major. $N$. Engl. J. Med, 343, 327-331 https://doi.org/doi:10.1056/NEJM200008033430503 (2000).

4. Barry, M. \& Sherlock, S. Measurement of liver-iron concentration in needle-biopsy specimens., 1, 100103 https://doi.org/doi:10.1016/s0140-6736(71)90838-5 (1971).

5. Bauman, J. H. \& Harris, J. W. Estimation of hepatic iron stores by vivo measurement of magnetic susceptibility. J. Lab. Clin. Med, 70, 246-257 (1967). 
6. Brittenham, G. M. et al. Magnetic-susceptibility measurement of human iron stores. N. Engl. J. Med, 307, 1671-1675 https://doi.org/doi:10.1056/NEJM198212303072703 (1982).

7. Carneiro, A. A. et al. An alternating current superconductor susceptometric system to evaluate liver iron overload. Rev. Sci. Instrum, 74, 3098-3103 https://doi.org/doi:10.1063/1.1570946 (2003).

8. Farrell, D. et al. Magnetic measurement of human iron stores. IEEE Trans. Magn, 16, 818-823 https://doi.org/doi:10.1109/TMAG.1980.1060810 (1980).

9. Fischer, R. et al. in Advances in Biomagnetism '91: Clinical Aspects (ed Hoke, M.)585-588(Elsevier, 1992)

10. Paulson, D. N., Fagaly, R. L., Toussaint, R. M. \& Fischer, R. Biomagnetic Susceptometer with Squid Instrumentation. IEEE Trans. Magn, 27, 3249-3252 https://doi.org/doi:10.1109/20.133904 (1991).

11. Avrin, W. F. \& Kumar, S. Noninvasive liver-iron measurements with a room-temperature susceptometer. Physiol. Meas, 28, 349-361 https://doi.org/doi:10.1088/0967-3334/28/4/002 (2007).

12. Marinelli, M. et al. Non-Invasive Measurement of Iron Overload in the Human Body. IEEE Trans. Appl. Supercond, 16, 1513-1518 https://doi.org/doi:10.1109/TASC.2005.869661 (2006).

13. Mueller, J. et al. Sensitive and non-invasive assessment of hepatocellular iron using a novel roomtemperature susceptometer. J. Hepatol, 67, 535-542 https://doi.org/doi:10.1016/j.jhep.2017.04.019 (2017).

14. Sheth, S. et al. Measurement of the liver iron concentration in transfusional iron overload by MRI R2* and by high-transition-temperature superconducting magnetic susceptometry. Clin. Imaging, 55, 6570 https://doi.org/doi:10.1016/j.clinimag.2019.01.012 (2019).

15. St Pierre, T. G. et al. Noninvasive measurement and imaging of liver iron concentrations using proton magnetic resonance., 105, 855-861 https://doi.org/doi:10.1182/blood-2004-01-0177 (2005).

16. Wood, J. C. et al. MRI R2 and R2* mapping accurately estimates hepatic iron concentration in transfusion-dependent thalassemia and sickle cell disease patients., 106, 1460-1465 https://doi.org/doi:10.1182/blood-2004-10-3982 (2005).

17. Fischer, R. in Magnetism in Medicine: a Handbook (eds. Andrew, M. \& Nowak, H.)286-301(Wiley$\mathrm{VCH}, 1998)$

18. Sharma, S. D., Hernando, D., Horng, D. E. \& Reeder, S. B. Quantitative susceptibility mapping in the abdomen as an imaging biomarker of hepatic iron overload. Magn. Reson. Med, 74, 673-683 https://doi.org/doi:10.1002/mrm.25448 (2015).

19. Barry, M. Liver iron concentration, stainable iron, and total body storage iron., $15,411-415$ https://doi.org/doi:10.1136/gut.15.5.411 (1974).

20. Pakbaz, Z. et al. Liver Iron Measurement by SQUID Compared to Liver Biopsy., 108, 3826-3826 (2006).

21. Butensky, E. et al. Variability in hepatic iron concentration in percutaneous needle biopsy specimens from patients with transfusional hemosiderosis. Am. J. Clin. Pathol, 123, 146-152 
https://doi.org/doi:10.1309/puuxegxdlh26nxa2 (2005).

22. Bush, V. J., Moyer, T. P., Batts, K. P. \& Parisi, J. E. Essential and toxic element concentrations in fresh and formalin-fixed human autopsy tissues. Clin. Chem, 41, 284-294 (1995).

23. Starr, T. N. et al. (eds) in Proceedings of the 12th International Conference on Biomagnetism.(eds. Nenonen, J., Ilmoniemi, R., \& Katila, T.) 986-989

24. Fung, E. B. et al. The new SQUID biosusceptometer at Oakland: first year of experience. Neurol. Clin. Neurophysiol. 2004, 5 (2004)

25. Sharma, S. D. et al. MRI-based quantitative susceptibility mapping (QSM) and R2* mapping of liver iron overload: Comparison with SQUID-based biomagnetic liver susceptometry. Magn. Reson. Med, 78, 264-270 https://doi.org/doi:10.1002/mrm.26358 (2017).

26. Fischer, R. in Metal Chelation in Medicine (ed. Crichton, R., Ward, R., \& Hider, R. C.) Ch. 7, 213-259 (Royal Society of Chemistry, 2017)

27. Overmoyer, B. A., McLaren, C. E. \& Brittenham, G. M. Uniformity of liver density and nonheme (storage) iron distribution. Arch. Pathol. Lab. Med, 111, 549-554 (1987).

28. Leung, N. W., Farrant, P. \& Peters, T. J. Liver volume measurement by ultrasonography in normal subjects and alcoholic patients. J. Hepatol, 2, 157-164 https://doi.org/doi:10.1016/s01688278(86)80074-5 (1986).

29. Engelhardt, R. et al. Agreement of liver iron quantification measurements with low Tc-SQUID biosusceptometers in Oakland, Torino and Hamburg. Int. Congr. Ser, 1300, 279-282 https://doi.org/doi:10.1016/j.ics.2007.01.048 (2007).

30. Rasmussen, S. N. Liver volume determination by ultrasonic scanning. Dan. Med. Bull, 25, 1-46 (1978).

31. Olivieri, N. F. \& Brittenham, G. M. Iron-chelating therapy and the treatment of thalassemia., 89, 739761 (1997).

32. Marinelli, M. et al. Total iron-overload measurement in the human liver region by the magnetic iron detector. IEEE Trans. Biomed. Eng, 57, 2295-2303 https://doi.org/doi:10.1109/TBME.2010.2053204 (2010).

33. Taylor, B. A. et al. Simultaneous field and R2 mapping to quantify liver iron content using autoregressive moving average modeling. J. Magn. Reson. Imaging, 35, 1125-1132 https://doi.org/doi:10.1002/jmri.23545 (2012).

34. Zuyderhoudt, F. M., Hengeveld, P., van Gool, J. \& Jorning, G. G. A method for measurement of liver iron fractions in needle biopsy specimens and some results in acute liver disease. Clin. Chim. Acta, 86, 313-321 https://doi.org/doi:10.1016/0009-8981(78)90387-x (1978).

35. Silver, M. M. et al. Perinatal hemochromatosis. Clinical, morphologic, and quantitative iron studies. Am. J. Pathol, 128, 538-554 (1987).

36. Bulte, J. W., Miller, G. F., Vymazal, J., Brooks, R. A. \& Frank, J. A. Hepatic hemosiderosis in non-human primates: quantification of liver iron using different field strengths. Magn. Reson. Med, 37, 530-536 
https://doi.org/doi:10.1002/mrm.1910370409 (1997).

37. Hackett, S., Chua-anusorn, W., Pootrakul, P. \& St Pierre, T. G. The magnetic susceptibilities of iron deposits in thalassaemic spleen tissue. Biochim. Biophys. Acta, 1772, 330-337

https://doi.org/doi:10.1016/j.bbadis.2006.12.007 (2007).

38. Ropert-Bouchet, M. et al. in First Congress of the International Biolron Society.

39. Xi, H. et al. A Room Temperature Ultrasensitive Magnetoelectric Susceptometer for Quantitative Tissue Iron Detection. Sci. Rep. 6, Article number: 29740, 1-7, doi:10.1038/srep29740 (2016)

\section{Figures}

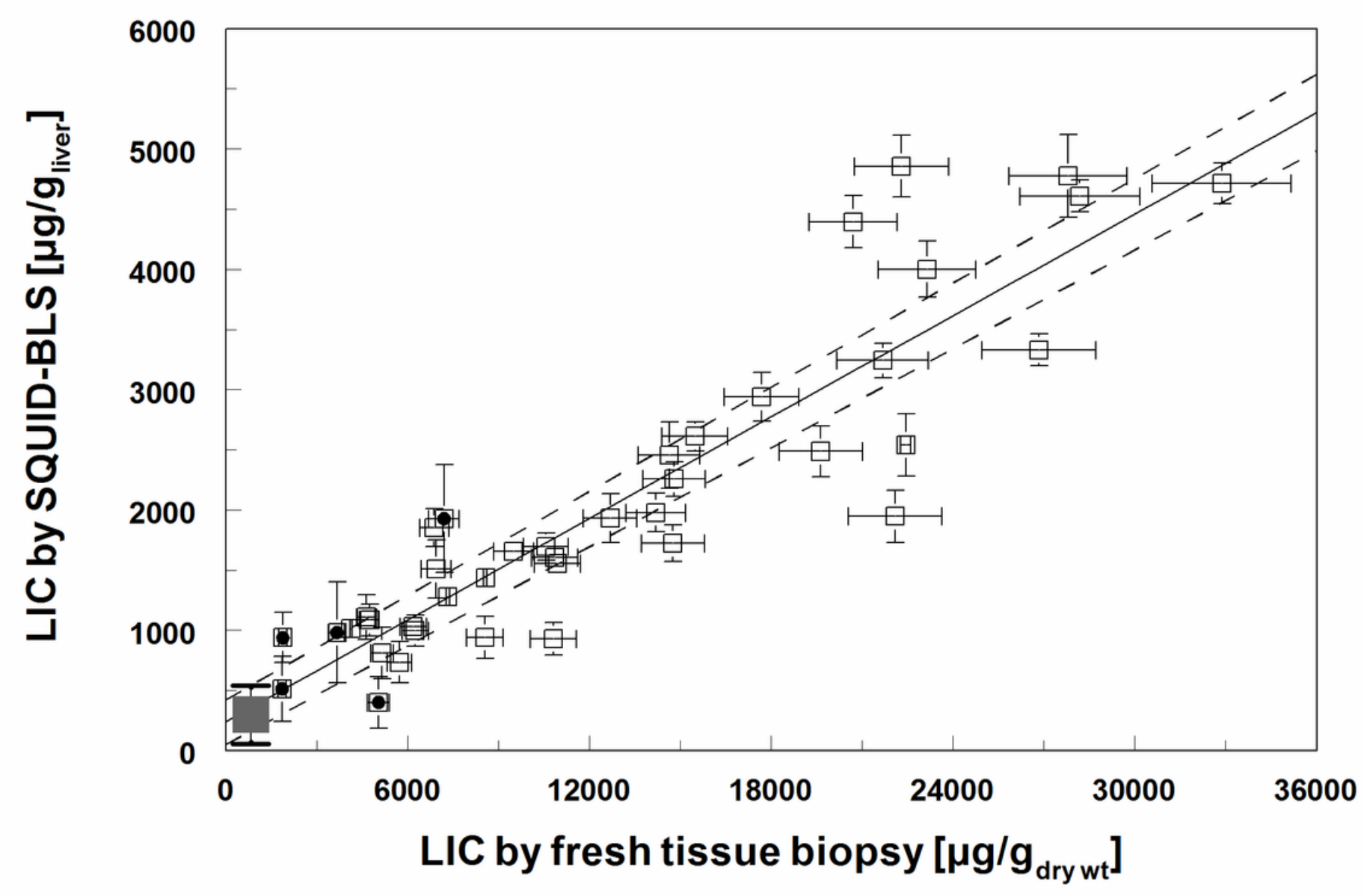

Figure 1

Comparison of Liver Iron Concentration by Analysis of Fresh Tissue Biopsy Samples and SQUID Biosusceptometry (BLS) Weighted regression analysis $(r 2=0.83)$ of liver iron measurements by SQUID biosusceptometry (LICBLS) versus LIC from heat-dried fresh tissue biopsies (LICFT) including 
uncertainties (squares) within 95\% confidence interval (dashed lines); solid circles indicate patients with $\mathrm{BMI}>30 \mathrm{~kg} / \mathrm{m} 2$, the normal range of LIC values is shown as large grey square.

\section{Figure 2}

Comparison of Liver Iron Concentration by SQUID biosusceptometry (BLS) and Analysis of Deparaffinized Biopsy Samples. For conversion of liver iron concentrations by SQUID biosusceptometry (BLS) into LIC from deparaffinized biopsy samples (rhomboids), a weighted linear fit (including uncertainties, $r 2=0.87$ ) resulted in a conversion factor of $6.60 \pm 0.25(\mu \mathrm{g} / \mathrm{gdry} \mathrm{wt}) /(\mu \mathrm{g} / \mathrm{gliver})$ : solid circles indicate SCD patients, the $95 \%$ confidence interval is shown by dashed lines.

\section{Figure 3}

Comparison of Liver Iron Concentration by SQUID biosusceptometry (BLS) and Total Iron Score of Histologic Samples. Nonlinear fit (solid blue line, $r 2=0.47$ ) of total iron score $(T I S)$ as exponential saturation function of liver iron measurements by biosusceptometry together with the $95 \%$ confidence interval (blue dashed lines). The black line represents TIS as function of LIC from paraffin-embedded biopsies published by Butensky et al21 and converted to LICBLS by 6.60 from Fig. 2 .

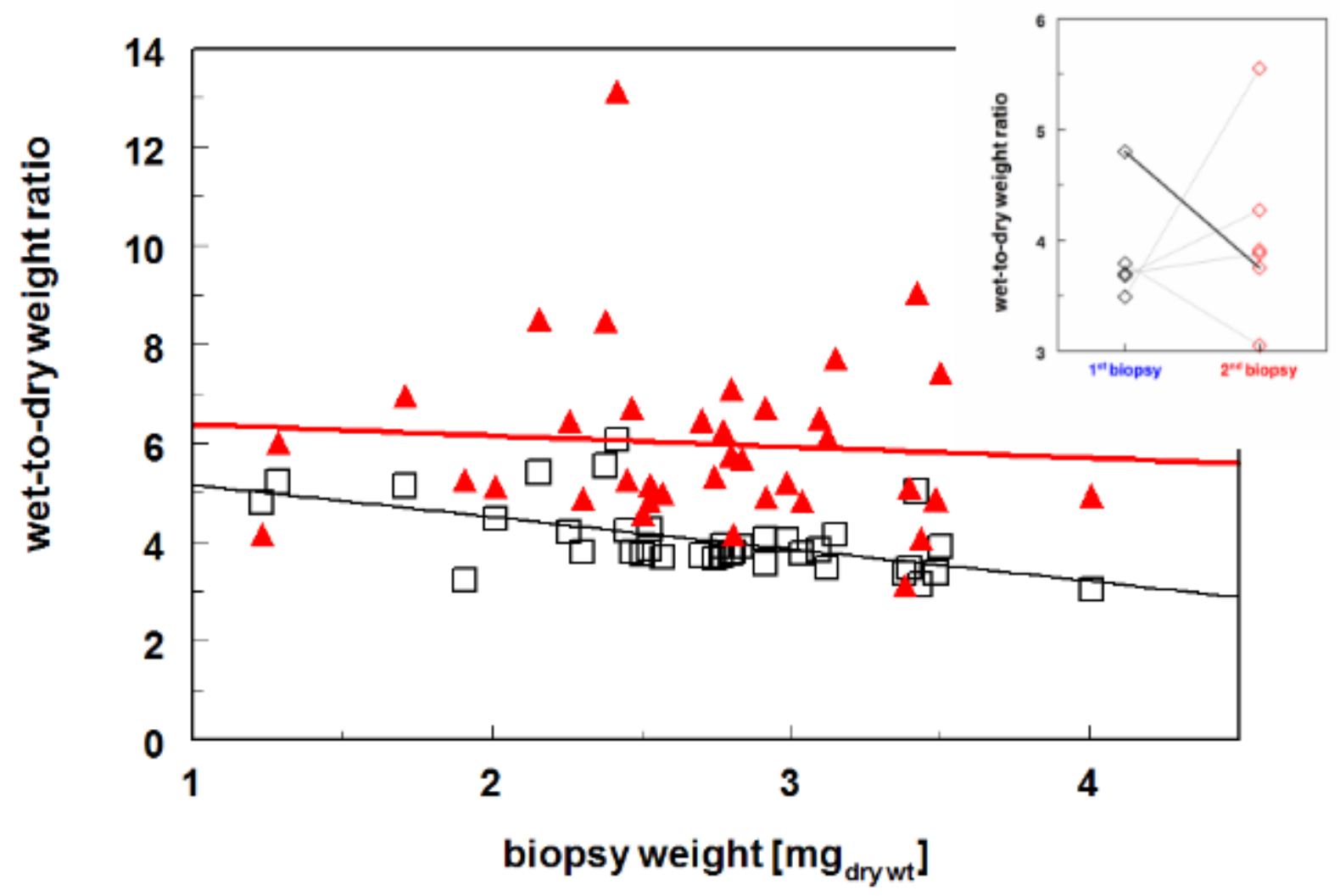




\section{Figure 4}

Relationship Between Biopsy Weight and Wet-to-dry Weight Ratio Without and With Adjustment for Hepatomegaly. Correlation between the wet-to-dry weight ratio (WDR, black squares) and dry weight of fresh tissue biopsies (black line: $r S=0.52, p<0.0001$ ). Taking the liver volume into account

(hepatomegaly), the adjusted ratio is indicated by red triangles resulting in a WDR* of $5.9 \pm 1.9$ (red line). The inserted scatter diagram shows the WDRs of repeated biopsies in 6 patients. 